\title{
Use of Mini-fragment Plates in Fracture Fixation
}

\author{
Vikas Kulshrestha ${ }^{1}$, Abin Stanley ${ }^{1}$, Santhosh Kumar ${ }^{1}$, Munish Sood $^{2}$
}

\section{Abstract}

Background: Over the last two decades, improved research in basic sciences has resulted in a better understanding of pathomechanics of skeletal injury. Today, we have modern implants and instrumentation which can allow fracture fixation while minimizing soft-tissue trauma. The use of mini fragment low profile locking plates is one such technique that is now increasingly being used to implement fragment specific fracture fixation in skeletal injuries.

Methods: At our level III military trauma center, we introduced the use of mini fragment plates in all types of fracture types in 2019 and over the next 12 months, we prospectively recorded data of 44 fracture cases of various long and small bones. We have used these plates with different objectives in various intra-articular, metaphyseal, or diaphyseal fractures in a manner to augment, supplement, or substitute the use of larger implants. We have described the exact method of use in each case.

Results: Out of 44 cases in 42 , we achieved fracture union with no loss of reduction and in one case there was a failure of fixation and in one case infected nonunion.

Conclusion: Mini-fragment plates are a very efficient tool available to carry out reduction and fixation of many fracture types and should be kept as a part of instrumentation and implant set for all cases of fracture fixation.

Keywords: Mini-fragment plates, fragment specific, fracture fixation.

\section{Introduction}

The rapid increase in the volume of skeletal trauma with its resulting socioeconomic impact has driven advancement in trauma care. Fracture fixation is as much an art as it is science. Fracture healing requires a complex interplay of biology and biomechanics, each helping the other. The surgeon cannot compromise one to optimize the other, making fracture fixation an extremely demanding procedure. It is training, experience, skill, and understanding which allows the surgeon to use both biological and biomechanical concepts to optimally fix skeletal injuries and ensure an ideal environment for bone healing. Fracture fixation

${ }^{1}$ Department of Orthopaedics, Command Hospital Air Force Station, Bengaluru, Karnataka, India,

${ }^{2}$ Department of Orthopaedics, INHS Asvini, Mumbai, Maharashtra, India

Address of Correspondence

Dr. Lt Col Munish Sood,

Department of Orthopaedics, INHS Asvini, Mumbai - 500 004, Maharashtra, India.

E-mail: soodmunishafmc@gmail.com techniques have evolved over the last century. With the use of lab models most skeletal injuries have been reclassified and newer fixation strategies have been advocated. At the same time with detailed knowledge of surgical anatomy and refinement of surgical skills, more surgical approaches have been identified to implement modern fracture fixation strategies. Till recently internal fixation of any fracture meant either plating or nailing. These two techniques were understood to be mutually exclusive. As the understanding of fracture fixation has evolved, it is now obvious that both techniques may be different in principles but they can be used together to provide better biomechanical constructs

Submitted Date: 15 July 2021, Review Date: 13 June 2021, Accepted Date: 16 Aug 2021 \& Published Date: 31 Dec 2021

Journal of Clinical Orthopaedics | Available on www.jcorth.com |DOI:10.13107/jcorth.2021.v06i02.441 This is an Open Access article distributed under the terms of the Creative Commons Attribution NonCommercial-Share Alike 4.0 License (http://creativecommons.org/licenses/by-nc-sa/4.0) which allows others to remix, tweak, and build upon the work non-commercially as long as appropriate credit is given and the new creation are licensed under the identical terms.

respecting biology. This is especially true for complex fracture patterns and metaphyseal fractures where a combination of nailing and plating may allow better construct strength and fracture healing environment. The concept of reduction plating of proximal tibial fracture before nailing was demonstrated by Benirschke et al. in 1995 [1]. Low profile mini-fragment locking plates have been available in the hand and foot implant portfolio but were rarely used for larger bones. However, it is only recently that surgeons have started using it in fracture fixation in various regions of the upper and the lower limb with different objectives [2]. These plates allow minimally invasive temporary reduction while the final plate or nail construct is developed to be left or removed at the end of the surgery. Frequently they are also being used as an adjunct, accessory, or alternate implant to allow minimal soft-tissue disruption 


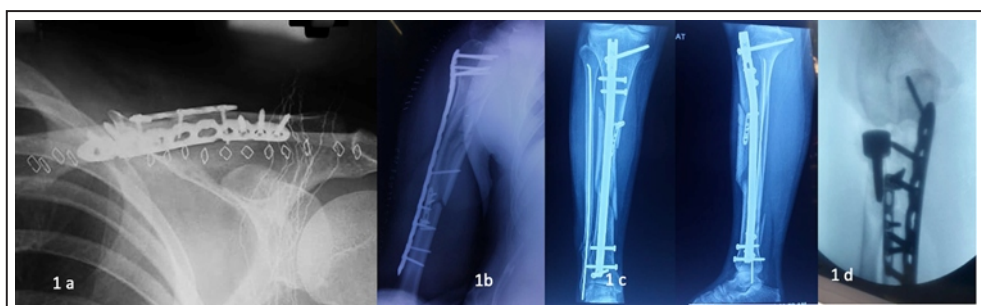

Figure 1: (a-d) The use of mini-fragment plates as an adjunct in fixation of comminuted fractures of clavicle, humerus, tibial shaft, and ulna.

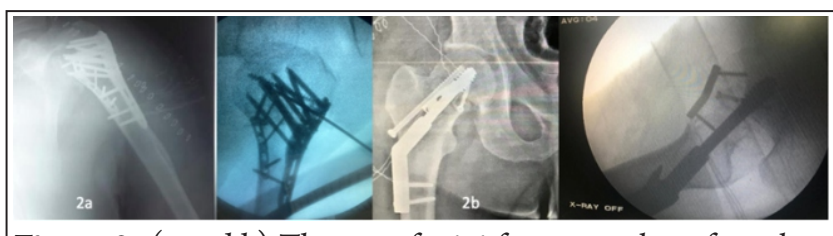

Figure 2: ( $a$ and b) The use of mini-fragment plates for calcar reconstruction in comminuted displaced fracture proximal humerus in 33-year-old male and in intracapsular neck of femur in 37-year-old male patient along with iliac crest graft.
[3]. Their use has minimized the soft tissue injury by avoiding the use of large clamps, or wire loops. Our study demonstrates the utility of minifragment plates in different fracture patterns in long and small bones.

\section{Methods}

This study was carried out at a military Level III trauma center working with a tertiary care referral hospital. We started using mini-fragment low profile locking plates for different fracture fixations since 2019. As the treatment offered to each patient is standard of care, no separate ethical approval was obtained. However, written informed consent was taken from all the patients. The $2.4 \mathrm{~mm}$ distal radius titanium locking plates and $2 \mathrm{~mm}$ locking plates from the handset were made available for all fracture fixation cases apart from the regular implants used for the surgery. We prospectively collected data on utilization of these minifragment plates in 44 cases of fracture fixation done over a 12-month period (2019-2020) in various case scenarios and fracture patterns (Table 1). All the surgeries were carried out by two trauma fellowship trained senior orthopedic surgeons. In this article, we describe the philosophy behind the use of the minifragment plates in these fracture fixation situations and their outcomes.

\section{Technique}

\section{Plating of multi-fragmentary} displaced diaphyseal fractures

Multi-fragmentary displaced fractures pose various challenges to fracture fixation. The fragments are small and with minimal soft tissue attachment, frequently they may be larger cortical bone with variable anatomy and multiple muscle attachments. Fixation of these fractures requires accuracy and diligence to restore alignment, optimize bony contact, neutralize muscular forces at the same time minimize soft-tissue dissection. We used locking plates for fixing these fractures aided by the use of a mini-fragment plate to achieve accurate butterfly fragment reduction and allow anatomical reduction of the primary fracture line without the use of bone clamps and multiple $\mathrm{K}$ wires. Bone clamps de-vascularize and $\mathrm{K}$ wires make it difficult to apply the final plate and, in both cases, still a separate interfragmentary fixation may be required which can risk communition and further devascularization. As against this, we used an extra periosteal minifragment plate with two non-locking screws to get fragment reduction which further was augmented with more locking screws if the plate is required to be left. The low profile of the plate allowed accurate contouring and minimized soft-tissue impingement while producing perfect fragment reduction. The mini-fragment plate allows the definitive implant to be used along with it. Fig. 1 demonstrates the use of mini-fragment plates in the fixation of comminuted fractures of the clavicle, humeral, and tibial shaft (Fig. 1).

\section{Calcar reconstruction of comminuted proximal humerus/femur fracture}

Four-part proximal humerus fractures are notorious for failing into varus and displaced medial metaphyseal spike predicts high failure. This mini-fragment plate comes to our rescue by allowing accurate reduction of the medial calcar and adjunct fixation in an extremely inaccessible area. The medial calcar arch of the humerus is deep even with the deltopectoral approach it is difficult to access and most of the time just allows a finger palpatory or preliminary $\mathrm{K}$ wire reduction before application of proximal humeral locking plates, which many times results in secondary displacement and failure. Mini-fragment plate with its extremely small footprint allows accurate and reasonably rigid fixation of the calcar preventing late collapse. Similarly in

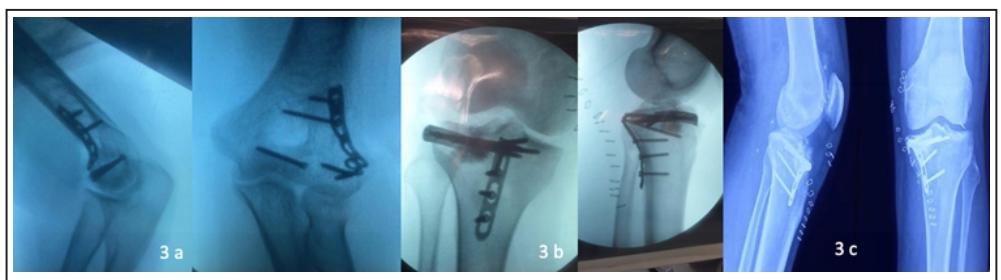

Figure 3: (a-c) Demonstrates the use of mini-fragment plates in the reconstruction of periarticular fractures of the distal humerus and rim fracture of the proximal tibia and reverse Segond injury.

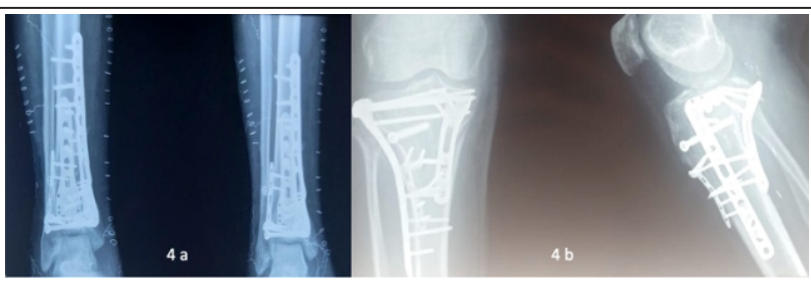

Figure 4: ( $a$ and $b$ ) Demonstrates the use of mini-fragment plates in the reconstruction of periarticular fractures of the distal tibia and proximal tibia. 


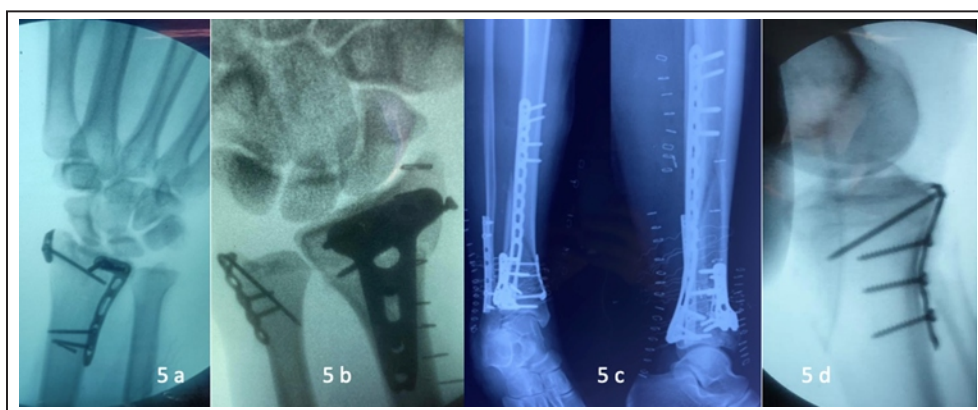

Figure 5: (a-d) Illustrates the use of mini-fragment plates to reattach ligament avulsions such as distal radioulnar joint (Triangular fibrocartilage complex; TFCC), Tillaux fracture ankle mortise (Anteroinferior tibiofibular ligament; AITFL), and knee joint (Posterior cruciate ligament; PCL).

comminuted displaced femoral neck fractures, miniplate helps reconstruct the calcar in a difficult to access zone of injury. In Fig. 2, we have elucidated the use of mini-fragment plates for comminuted displaced fracture proximal humerus and intracapsular neck of femur (Fig. 2).

\section{Complex articular reconstructions}

Small fragment plates are extremely important in the fixation of small critical fracture fragments in a setting of complex articular fractures. They allow piece by piece reconstruction of the jigsaw puzzle thus ensuring adequate reduction and reconstruction of the subarticular surface even permitting the use of bone graft and its substitutes. Frequently the intraarticular fractures are multi-fragmentary with small fracture fragments almost completely covered with articular surface. This allows a very small surface for use of implants. The use of minifragment plates allows good buttressing of these fragments along with

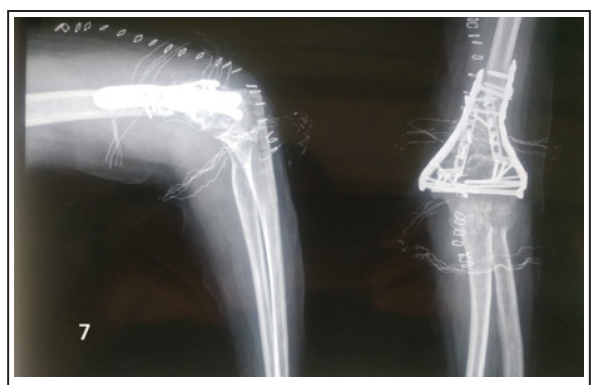

Figure 7: Nonunion of distal humerus fracture revised using fixed orthogonal fixation and grafting in a 42-year-old male. interfragmentary fixation while avoiding the articular surface encroachment and impingement. Sometimes the articular fracture is rim avulsion fracture especially in the knee and they are like capsuloligamentous avulsions. These fractures have small bony fragments and they need rim reconstruction using minifragment plates. Figs. 3 and 4 demonstrate the use of mini-fragment plates in periarticular reconstruction.

\section{Reattachment of bony ligamentous avulsions}

Frequently in multi-fragmentary fractures, there could be a small bony avulsion of the ligament from its attachment and its reattachment becomes critical to the stabilization of the articular mortise. While the primary fracture implant fixes the major fracture line it is not able to fix the avulsed ligament. The use of mini-fragment plates using the tension band principle solves the problem. Fig. 5 illustrates the use of mini-fragment plates to reattach ligaments to stabilize distal radioulnar joint (triangular fibrocartilage complex), ankle mortise (anteroinferior tibiofibular ligament [AITFL]), and knee joint (posterior cruciate ligament [PCL]) (Fig. 5).

Reconstruction of metaphyseal injuries of long bone using a hybrid nail plate construct

Lower limb long bone fractures are preferably nailed to allow early weight bearing as they are load sharing device. However, in the metaphyseal area where the medullary canal flares out, the use of intramedullary nail does not ensure proper fracture reduction. The use of hybrid nail plate constructs can combine the advantages of plating in allowing accurate reduction of metaphyseal fractures while nailing is performed. The plate here could be used as a temporary reduction tool or could be left as an adjunct fixation to enhance the stability of the construct. Fig. 6 shows the use of mini-fragment plate as reduction plate in metaphyseo-diaphyseal tibial fracture (Fig.6).

\section{Providing orthogonal fixation improving the rigidity of fixation in non-unions}

Fracture non-unions could be due to failure of biology or the biomechanics. In cases where fracture stability has been a concern due to poor biomechanical reconstruction of the skeletal injury, we see hypertrophic non-unions and these require refixation with an improved multiplanar reconstruction. Minifragment plates come to the rescue in these cases especially where fracture fragments are small and allow only limited area for use of implant without impingement. One such area is the elbow with nonunion of the supracondylar humerus fracture. The use of these additional mini-fragment plates allows 
orthogonal plate-plate reconstruction of both radial and ulnar pillars. They can be used to avoid the critical articular zones and fossa thus allowing elbow movements without impingement. Fig. 7 shows one such case of nonunion of distal humerus fracture revised fixed orthogonal fixation and grafting (Fig. 7).

\section{Use in small plates in place of wiring or lag screws}

Till recently most of the small bone fractures such as the patella, scaphoid, and radial head were fixed either with screws or wires using cerclage, tension band, or simple interfragmentary lag technique. We have used mini-fragment plating for these fractures in the setting of communition or neglected injuries with nonunion and poor bone stock with good results. The use of multiple plates in these bones allow orthogonal fixation and provide multiplanar stability. Fig. 8 shows 4th months old ununited displaced fracture patella, a fresh radial head comminuted fracture, and a recalcitrant nonunion of scaphoid reconstructed using mini-fragment plates (Fig. 8).

\section{Results}

At 1 year of follow-up out of 44 cases in 42 cases fracture reduction was maintained and fracture healed uneventfully. In one case of femoral neck fracture where calcar was deficient and grafted the fixation failed at 6 months as the patient being alcohol dependent did not comply with weight-bearing restriction and underwent revision to Total Hip Replacement. In one case of comminuted open tibial plateau fracture, there was delayed union with the implant infection and at 6 months needed debridement removal of implant and external fixation.

\section{Discussion}

Improved understanding of pathomechanics of fractures has changed the management of skeletal injuries. Over the last two decades, newer surgical approaches have evolved to implement newer biomechanical fixation strategy. The implant industry has responded by improving design, metallurgy, and instrumentation used to fix fractures. Today, most acute skeletal injuries requiring fixation are accurately reduced to restore alignment and rotation. Articular surfaces are diligently reconstructed. To achieve these goals more and more fragment specific fixation is being resorted to in a biologically friendly manner and mini-fragment plates have provided the necessary tool to implement the new concept effectively [4]. These strategies have been well tested in labs and are now available for clinical use requiring evaluation [5]. Our article has attempted to highlight the growing usage of this implant in our practice wherein 1 year at our thus bringing out the modality of usage and the advantages of the same.

The use of mini-fragment plates for fragment specific fracture fixation for distal radial fractures was introduced by Medoff in his paper published in 1998 [6]. His description of the technique and usage of various implants was in great details, however with lack of expertise and limited availability of specialized implants and instruments the

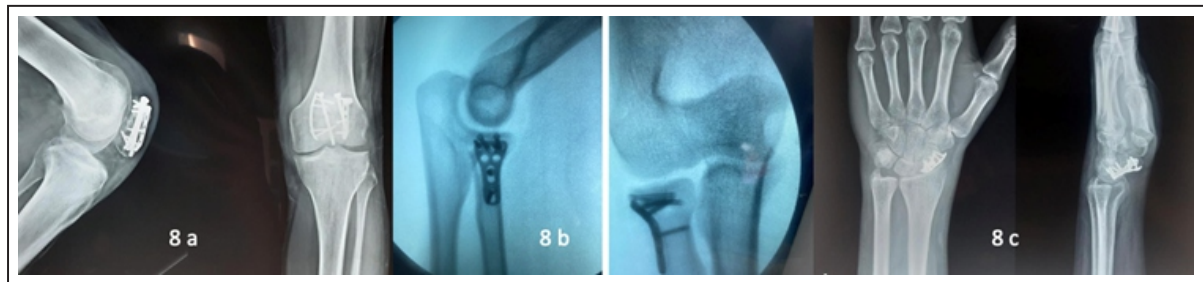

Figure 8: (a-c) Fourth months old ununited displaced fracture patella, a fresh radial head comminuted fracture, and a recalcitrant nonunion of scaphoid reconstructed using minifragment plates. complication rates were high. It is only recently that with the understanding of techniques and better availability of implants and instruments more surgeons are using fragment specific fixation strategies now in more than one type of fractures [2].

Very similar to our technique wherein we used mini-fragment plates to reduce and unitize the butterfly fragment of a comminuted midshaft displaced clavicle fracture before definitive plating Bishop and Castillo published their similar work [7]. While managing comminuted intraarticular distal radial fractures, we performed preoperative CT scan in all cases and used technique as described by Hozack and Tosti [8] in performing fragment specific fixation. In most cases, we used a temporary distractor to disimpact the fracture and hold it. We fine-tuned the reduction by volar and occasional dorsal approach and used mini-fragment plates to stabilize volar and dorsal ulnar fragments, radial styloid fragment, and occasional dorsal wall fragment. Our low-profile internal fixation allowed immediate finger movements and early forearm rotations. In all cases, we splinted the wrist in a brace for 6 weeks. In the upper limb distal

\begin{tabular}{|c|c|}
\hline \multicolumn{2}{|l|}{ Table 1: Various fractures fixed using mini- } \\
fragment plates \\
\hline Fracture & $\begin{array}{c}\text { No. of } \\
\text { patients }\end{array}$ \\
\hline Clavicle & 3 \\
\hline Proximal humerus & 1 \\
\hline Shaft humerus & 1 \\
\hline Distal humerus & 2 \\
\hline Proximal forearm & 4 \\
\hline Distal radius & 8 \\
\hline Scaphoid & 1 \\
\hline Pelvis & 2 \\
\hline Neck of femur & 1 \\
\hline Patella & 1 \\
\hline Tibial plateau & 6 \\
\hline Proximal third shaft tibia & 1 \\
\hline Mid shaft tibia & 1 \\
\hline Distal third tibia & 2 \\
\hline Tibial plafond & 6 \\
\hline Ankle & 2 \\
\hline Misc. & 2 \\
\hline Total & 44 \\
\hline
\end{tabular}

(C) | Journal of Clinical Orthopaedics | Published by Orthopaedic Research Group | Volume 6 | Issue 2 | July-dec 2021 | Page 05 
radius fractures especially the metaphyseal fractures including those with intra-articular extensions pose a significant challenge to secure fixation, we used mini-fragment plates in an orthogonal manner along with larger anatomical locking plates to fix some of these injuries helping us avoid impinging onto the fossa around the elbow and the articular surfaces while significantly improving rigidity of the construct, very similar to the study published by Russell et al. [9]. We also used these plates as a primary fixation for carpal fixation such as scaphoid nonunion, ulnar styloid avulsions, capitellum fractures, and comminuted radial head fractures.

Similar to the upper limb injuries our usage of mini-fragment plates for the lower limb fractures was quite varied. It was extensively used for fixation of ligamentous avulsions like PCL, AITFL, and posteroinferior tibiofibular ligament as part of pilon fractures and patellar tendon avulsion as part of plateau fracture. As described by Berinschke, we used it as a reduction plate on more than one occasion in proximal and distal tibial nailing. With evolving concepts of proximal tibial fractures a great deal of emphasis has been laid in constructing columns around the condyles. Furthermore, with the application of relatively unfamiliar approaches, positions (prone/ lateral decubitus) and difficult intra-op imaging, particularly for posteromedial or posterolateral condyles, anterior tibial tubercular fragments, rim avulsions, etc., provisional fixation takes precedence in obtaining meta-diaphyseal alignment before definitive fixation with newer low profile $3.5 \mathrm{~mm} / 4.5 \mathrm{~mm}$ plates. Work from Bogdan and Tornetta emphasized on advantages of lesser prominent hardware, malleability for contouring, multiple points fixations and also the ability to capture smaller fragments using smaller and more clustered screw options. We explored these advantages effectively in fixing Pilon fractures in our center and found them to be extremely beneficial to minimize damage to soft tissues, preserve vascularity while achieving near anatomical reduction of escape fragments such as Wagstaff's, Tillaux fracture, and other comminuted components of Pilon fracture. Similar, implications were employed, efficacy, and safety of MFP techniques in tibial plafond fracture are endorsed by Dang et al. in their series of 37 cases of Pilon fractures treated with a particular miniplating system [10]. Gentile et al. [11] demonstrated the equivalent results of the use of mini-fragment plates in fixation of distal fibula as compared to traditional $3.5 \mathrm{~mm}$ plating systems. We used mini-fragment plates in the fibula in addition to $3.5 \mathrm{~mm}$ fixation especially to fix the Wagstaff fragment which was associated with the primary fracture line. Our study is unique as it is one of the first to bring forth such extensive successful usage of mini-fragment plates in varied regions of the body using different fracture fixation principles. It strongly supports the usage of mini-fragment plates in a minimal invasive fracture fixation strategy avoiding the use of large clamps and reduction tools requiring extended exposure and soft tissue dissection. The limitation is that being a safety and efficacy type of study although we looked at outcomes in terms of fracture healing and complications such as loss of reduction, delayed union, nonunion, and implant infection, we did not compare the functional outcomes with standard plating and nailing techniques. As we have successfully demonstrated the possibility of safe and effective usage of these implants in the fixation of fractures of varied pattern, it can form the basis for implementing stronger comparative clinical trials to document the advantages of this fracture fixation technique.

\section{Conclusion}

Mini-fragment plates are a very efficient tool available to carry out reduction and fixation of many fracture types and should be kept as a part of instrumentation and implant set for all cases of fracture fixation.

\section{Clinical Relevance}

Role of mini-fragments plates can form the basis of fixation of fractures techniques especially comminuted fractures.

Declaration of patient consent: The authors certify that they have obtained all appropriate patient consent forms. In the form, the patient has given his consent for his images and other clinical information to be reported in the Journal. The patient understands that his name and initials will not be published, and due efforts will be made to conceal his identity, but anonymity cannot be guaranteed.

Conflict of Interest: NIL; Source of Support: NIL

\section{References}

1. Benirschke SK, Henley MB, Ott JW. Proximal one-third tibial fracture solutions. Orthop Trans 1995;18:1055-6.

2. Archdeacon MT, Wyrick JD. Reduction plating for provisional fracture fixation. J Orthop Trauma 2006;20:206-11.

3. Bogdan Y, Tornetta III P. Fragment-specific fixation of proximal tibia fractures: Case report and surgical technique. J Orthop
Trauma 2018:e1-5 DOI: 10.1097/BOT.0000000000001253

4. SadekAF, Ahmed MA, Kader MA, El-Shafie MB. Lateral condylar retrograde humeral nail for management of high-energy distal h u m e ral fractures. J Orthop Surg 2019;27:2309499019847922.

5. Knox R, Curran P, Herfat S, Kandemir U, Marmor M. The 
influence of mini-fragment plates on the mechanical properties of long-bone plate fixation. OTA Int 2019;2:e034.

6. Medoff RJ. Immediate internal fixation and motion of comminuted distal radius fractures using a new fragment specific fixation system. Orthop Trans 1998;22:165.

7. Bishop JA, Castillo TN. Provisional mini-fragment plate fixation in clavicle shaft fractures. Am J Orthop (Belle Mead NJ) 2013;42:470-2.

8. Hozack BA, Tosti RJ. Fragment-specific fixation in distal radius fractures. Curr Rev Musculoskelet Med 2019;12:190-7.
9. Russell GV Jr., Jarrett CA, Jones CB, Cole PA, Gates J. Management of distal humerus fractures with minifragment fixation. J Orthop Trauma 2005;19:474-9.

10. Dang KH, Ornell SS, Huynh RA, DeLeon JC, Pesek R, Karia RA. Early clinical and radiographic outcomes of a minifragment, low profile plating system in tibial plafond fractures. Injury 2019;50:1773-80.

11. Gentile J, Taylor BC, Chan R, French B. Clinical comparison of minifragment plates versus conventional semitubular plates for fixation of distal fibula fractures. HSS J 2015;11:148-53.
Conflict of Interest: NIL

Source of Support: NIL
How to Cite this Article

Kulshrestha V, Stanley A, Kumar S, Sood M. Use of Mini-fragment Plates in Fracture Fixation. Journal of Clinical Orthopaedics July-Dec 2021;6(2):2-7. 